[zur Veröffentlichung in Studia Linguistica XX/2001, Breslau Acta Universitatis]

Gisela Zifonun, IDS Mannheim

\title{
Grammatik des Deutschen im europäischen Vergleich
}

\section{Vorbemerkung, Verortung in der Tradition}

In der Abteilung Grammatik des Instituts für Deutsche Sprache, Mannheim, wird derzeit ein neues Projekt entwickelt, und zwar das einer Grammatik des Deutschen im europäischen Vergleich (GDE). Dieses Projekt fügt sich ein in die kontrastive Tradition des IDS, ist jedoch andererseits auch in vieler Hinsicht innovativ.

Bevor ich das Projekt im Einzelnen vorstelle, versuche ich den Bogen zurück zu den kontrastiven Grammatiken zu schlagen.

Gerade die Leserschaft polnischer Germanisten braucht an die Tradition kontrastiver Grammatikschreibung sicher nicht eigens erinnert zu werden. Denn diese Tradition, die untrennbar mit dem Namen Ulrich Engel verknüpft ist, ist gerade erst in der neu erschienenen deutsch-polnischen kontrastiven Grammatik kulminiert. Im Bereich der kontrastiven Grammatiken zu Sprachenpaaren, von denen das Deutsche ein Element ist, verfügt das IDS also über eine vergleichsweise reiche Tradition. Am IDS oder in Kooperation mit dem IDS wurden kontrastive Grammatiken zu den Sprachenpaaren Deutsch - Französisch (Zemb 1978), Deutsch - Serbokroatisch (Engel/Mrazović 1986), Deutsch - Spanisch (Cartegena/Gauger 1989), Deutsch - Rumänisch (Engel u.a. 1993) erarbeitet. Zum Sprachenpaar Englisch - Deutsch liegt mit Hawkins 1986 eine typologisch-vergleichende Grammatik vor. Die deutsch-polnische kontrastive Grammatik, die unter der Leitung von Ulrich Engel erarbeitet wurde, ist 1999 erscheinen. Abraham 1994 und Glinz 1994 konfrontieren das Deutsche, mit durchaus unterschiedlicher Akzentsetzung, mit mehreren anderen europäischen Sprachen. An der Berliner Humboldt-Universität laufen derzeit die Vorarbeiten zu einer deutsch-russischen kontrastiven Grammatik (Initiative Wolfgang Gladrow und Michail Kotin). Die Aufgabe einer 'Grammatik des Deutschen im europäischen Kontext' ist also hinlänglich vorbereitet.

\section{Projektfakten und -daten}

Das Projekt wurde Ende 1998 mit zunächst nur zwei Mitarbeiterinnen begonnen. Vorgesehen ist eine verlängerte Konzeptions- und Explorationsphase, mit der der Neuartigkeit der 
Aufgabenstellung Rechnung getragen werden soll. In der eigentlichen Arbeitsphase ist an einen festen Mitarbeiterstab von vier bis fünf Wissenschaftlern gedacht, der durch Kooperationen und befristete Projekte (etwa im Rahmen von Dissertationen oder Gastaufenthalten) verstärkt werden soll.

Die globale Aufgabenstellung ist: Das Deutsche soll sprachtypologisch verortet und mit mehreren europäischen Sprachen kontrastiert werden. ${ }^{1}$ Geplant ist eine eingehendere Kontrastierung mit folgenden Sprachen: Niederländisch, Englisch, Französisch, Spanisch, Polnisch, sowie Ungarisch und/oder Türkisch. Neben diesen Kontrastsprachen im engeren Sinne werden weitere europäische Sprachen, z.B. skandinavische, keltische oder kaukasische Sprachen, bei der Überblicksdarstellung einzubeziehen sein; auch im Detail können andere europäische Sprachen berücksichtigt werden, wenn es sich vom grammatischen Phänomen her anbietet. ${ }^{2}$ Bei der Wahl der Kontrastsprachen spielen unterschiedliche Gesichtspunkte eine Rolle: Zum einen sollen unterschiedliche Sprachgruppen innerhalb und außerhalb der indoeuropäischen Sprachfamilie und unterschiedliche areale Bereiche berücksichtigt werden. Zum anderen aber ist auch die Bedeutung des Deutschen als Lernersprache ein Faktor: Sprachgebiete, in denen das Deutsche intensiv gelehrt und gelernt wird, wie die Länder Mittel- und Osteuropas, sollen besonders berücksichtigt werden. Selbstverständlich wäre es wünschenswert und käme der wissenschaftlichen Ausgewogenheit zugute, wenn so viele Kontrastsprachen wie möglich einbezogen würden. Letztlich jedoch schränkt die Frage der Machbarkeit die Umsetzung dieser Wunschvorstellung ein und es erscheint ratsam - auch im Interesse einer möglichst gründlichen Einzelkontrastierung -, es bei einer guten Handvoll von Kontrastsprachen zu belassen.

Zunächst wurden Pilotstudien zum Genus des Substantivs und zu den Pronomina sowie zum Relativsatz erarbeitet. Die Projektkonzeption sowie die Pilotstudien wurden bei einem Kolloquium mit Fachkollegen aus den Bereichen germanistische Grammatikschreibung, kontrastive Grammatik, Auslandsgermanistik, Deutsch als Fremdsprache und Sprachtypologie im November 1999 diskutiert. Mit der positiven Einschätzung des Projekts ist der Startschuss für eine intensivere Projektarbeit gegeben. Dies bedeutet auch, dass neue Mitarbeiter mit ergänzenden Sprachkompetenzen hinzugewonnen werden müssen.

\footnotetext{
1 Zur aktuellen typologischen Forschung zum Deutschen vgl. den Sammelband Lang/Zifonun 1996, darin insbesondere Lang 1996.

${ }^{2}$ Die Kontrastsprachen und das Deutsche zusammen werden als 'Vergleichssprachen' bezeichnet.
} 


\section{Projektziele}

a) wissenschaftsintern: Fokussierung, Vereinheitlichung der Kategorisierungen und der Terminologie

Die vorliegenden wissenschaftlichen Grammatiken des Deutschen, einschließlich der „Grammatik der deutschen Sprache“ (GDS) (vgl. Zifonun/Hoffmann/Strecker u.a. 1997) enthalten kaum explizite Angaben zur sprachtypologischen Einordnung des Deutschen. Der typologische Vergleich sollte aber - so zeigt die neuere Forschung - eine wichtige Dimension auch der einzelsprachlichen Grammatikographie sein. Die spezifischen Formen und Konstruktionen, mit denen eine Einzelsprache grammatische Funktionen realisiert, können hier vor dem Hintergrund der universalen Möglichkeiten und des Spektrums sprachlicher Variation abgehoben werden. Ihre Eigenart und ihre charakteristische Kontur treten dadurch deutlicher und prägnanter hervor, als dies bei einer isolierten Betrachtungsweise überhaupt möglich ist. In den letzten Jahren wurde vor allem im Rahmen des EUROTYP-Projektes die typologische Erforschung der europäischen Sprachen erheblich vorangetrieben. ${ }^{3}$

Dabei sind selbstverständlich auch wichtige Bausteine zur typologischen Beschreibung des Deutschen erarbeitet worden. Eine geschlossene Gesamtdarstellung der „Eurotypologie“ des Deutschen, also eine typologische Verortung des Deutschen im Rahmen der europäischen Sprachen, ist damit jedoch noch nicht geleistet. Diese Lücke soll das vorliegende Projekt schließen.

In methodischer Hinsicht betrachten wir in erster Linie den mit dieser Herangehensweise verbundenen Fokussierungseffekt als gewinnbringend: Ein Gegenstand X, hier die Grammatik des Deutschen, ist klarer und schärfer zu erkennen, wenn nicht nur beschrieben wird, wie $\mathrm{X}$ ist (siehe GDS), sondern $\mathrm{X}$ auf der Folie der Möglichkeiten, anders zu sein, charakterisiert wird. Das Deutsche ist der 'Vordergrund', der sich auf dem Hintergrund der grammatischen Optionen der europäischen Sprachen abhebt.

Ein sekundärer wissenschaftsinterner Nutzen des Unternehmens sollte in der sprachübergreifenden Vereinheitlichung grammatischer Kategorisierung und Begrifflichkeit bestehen. Zum Teil ohne Not divergierende Begrifflichkeit, wie sie in den Standardgrammatiken europäischer Sprachen häufig festzustellen ist, kann den Blick auf

\footnotetext{
${ }^{3}$ Das Projekt "Typology of Languages in Europe" wurde im Projektzeitraum 1990-1994 von der ESF gefördert. Geplant sind beim Verlag de Gruyter, Berlin, EUROTYP-Veröffentlichungen zu insgesamt neun Bereichen: Konstituentenordnung, Aktanz und Valenz, Adverbialkonstruktionen, Wortprosodische Struktur, Syntax der Klitika, Tempus und Aspekt, NP-Struktur, Diskurspragmatik, Subordination und Komplementation. Die sechs erstgenannten Publikationen sind bereits erschienen.
} 
Gemeinsamkeiten verstellen (vgl. König 1996, Willems 1999). Ein Paradebeispiel ist dabei die Kategorienbildung im Bereich der zentralen grammatischen Funktionen bzw. der Satzglieder. Hier stoßen einerseits die traditionellen Terminologien (etwa mit Subjekt, Akkusativobjekt) und die moderne z.B. dependenz- und valenzgrammatische Terminologie (etwa mit Nominativergänzung, Akkusativergänzung) aufeinander. Andererseits aber wird eine einheitliche Terminologie auch durch "echte" Unterschiede zwischen den Sprachen erschwert. In der Mehrzahl der westeuropäischen (germanischen und romanischen) Sprachen gibt es nur noch rudimentäre Kasusunterscheidungen. Daher wird z.B. bei der Beschreibung des Norwegischen oder Französischen nicht von Objekten in einem bestimmten Kasus gesprochen, sondern von direktem Objekt, indirektem und ggf. obliquem oder präpositionalem Objekt. Allein schon die unterschiedliche Anzahl der Grundunterscheidungen macht hier eine wechselseitige Zuordnung der Kategorien schwierig: Orientiert man sich z.B. an den morphosyntaktisch unterschiedenen Kasus, so wird man im Deutschen traditionell Subjekt und drei Kasusobjekte sowie das Präpositionalobjekt differenzieren. Gegenüber dieser Einteilung in fünf Kategorien liefert die andere Herangehensweise zunächst nur vier Kategorien. Damit habe ich, wie klar sein dürfte, nur eine vergleichsweise einfache Schwierigkeit angedeutet, die der Sprachwirklichkeit nicht gerecht wird. Die valenzgrammatische Tradition hat ja gezeigt, dass deutlich mehr "Ergänzungsfunktionen" zu unterscheiden sind: So unterscheidet Engel in seiner "Deutschen Grammatik" (Engel 1991) 11 Ergänzungsklassen. Diese werden in Engel u.a. 1999 für das Polnische übernommen, jedoch um eine weitere Klasse "Intrumentalergänzung" erweitert. Auch wenn die Definition der Ergänzungsklassen nicht direkt auf den morphologischen Kasus beruht, so spiegelt sich doch zumindest indirekt hier das gegenüber dem Deutschen umfangreichere Kasussystem des Polnischen wider. Wenn schon bei zwei Sprachen wie Polnisch und Deutsch, die immerhin noch ein mehrgliedriges Kasussystem haben, solche Divergenzen auftreten, wie kann der ganzen Bandbreite vom Englischen oder Norwegischen (Kasusdifferenzierung im ZweiKasus-System nur bei Pronomina) bis zum Ungarischen (24 Kasus nach Lotz 1939, 18 Kasus nach Kiefer 1987) Rechnung getragen werden? Hier stellt sich eine Aufgabe, die weit über das rein Terminologische hinausgeht, die aber, wenn sie gelöst werden kann, auch zu einer Bereinigung der Terminologien führen kann.

b) anwendungsbezogen/wissenschaftspolitisch: Interface zwischen wissenschaftlicher deutscher Grammatik und Grammatik für Lerner mit bestimmten Ausgangssprachen

Wenn das Deutsche sich als Sprache von einigem Gewicht in Europa (als geographisches und politisches Gebilde) behaupten soll (Deutsch als 'Verkehrssprache in Europa'), müssen die 
Lehr- und Vermittlungsbedingungen für Deutsch als Fremdsprache verbessert werden. Unser Projekt ist im Zusammenhang zu sehen mit der grammatikographischen Aufbereitung des Wissens speziell für den Adressatenkreis der Dozenten und Studierenden des Fachs Deutsch/Germanistik an ausländischen Hochschulen. Zwar existieren - neben einer Reihe kontrastiver Grammatiken 'deutsch-X' im engeren Sinne - sowohl von deutschen Wissenschaftlern geschriebene Grammatiken, die sich als Grammatiken für Deutsch als Fremdsprache verstehen (Helbig/Buscha, Schulz-Griesbach, z.T. auch Engel), als auch eine große Zahl jeweils in der Lernersprache geschriebener deutscher Grammatiken z.B. für den norwegischen oder polnischen Lerner. Die besondere Eignung solcher Grammatiken für den Ausländerunterricht beruht in der Regel auf gediegener empirischer Erfahrung in der Vermittlung des Deutschen allgemein und auf der erfahrungsgestützten Auswertung von jeweils bilateralen Gemeinsamkeiten und Unterschieden zwischen dem Deutschen und der Muttersprache der Lerner. Ein Kompendium, das eine theoretisch abgesicherte Bestandsaufnahme über alle gegebenenfalls $\mathrm{zu}$ berücksichtigenden und besonders $\mathrm{zu}$ gewichtenden Besonderheiten des Deutschen bereitstellen würde, existiert aber bislang nicht. Diese Lücke könnte durch unser Vorhaben geschlossen werden: GDE als Interface zwischen deskriptiver Grammatik des Deutschen (z.B. GDS) und spezifischen Deutsch-Grammatiken für Lerner bestimmter Sprachen:

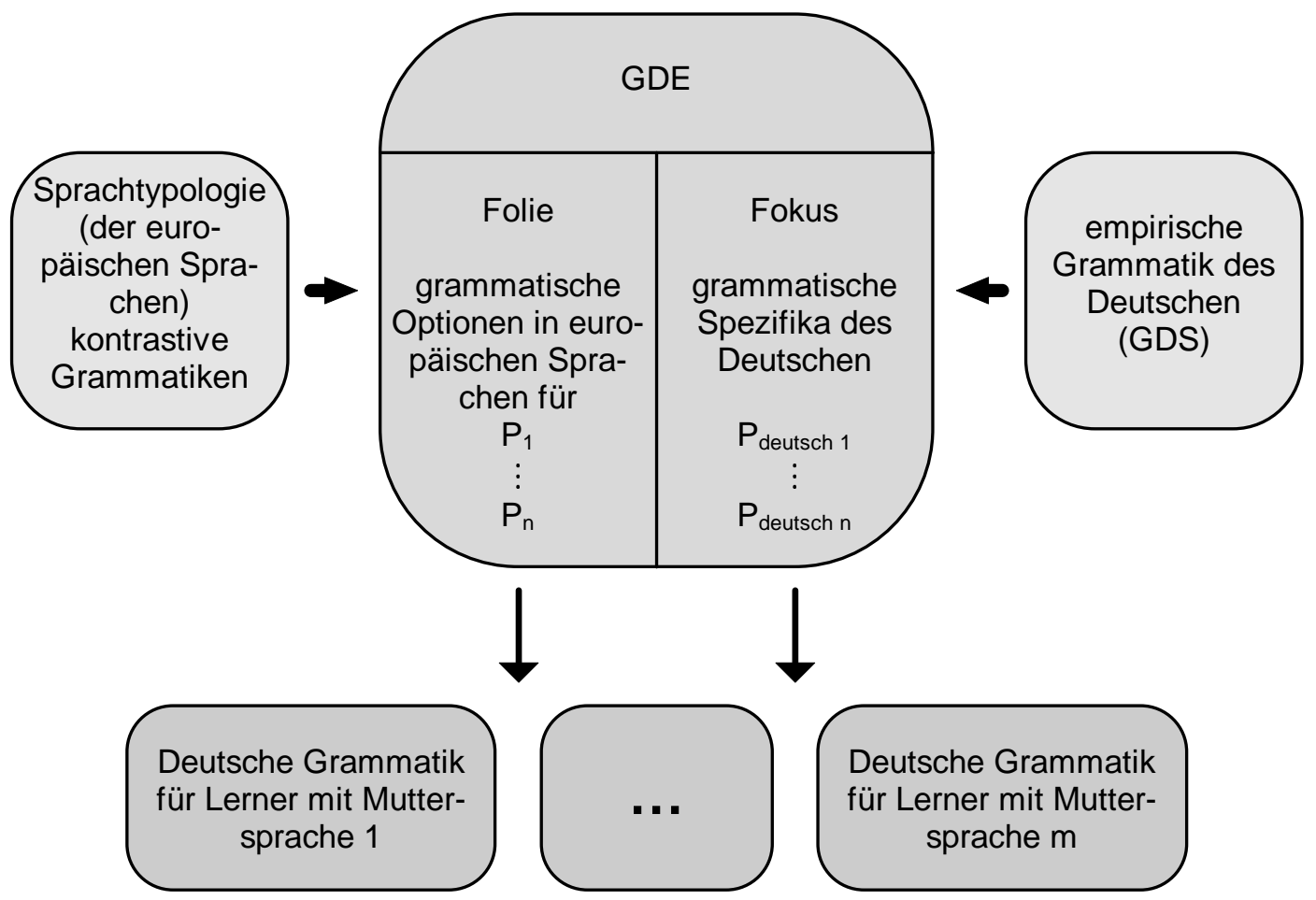


Die GDE schöpft somit aus zwei Quellen: zum einen aus der Grammatikographie des Deutschen, zum anderen aus der sprachtypologischen und kontrastiven Forschung. Ihre spezifische Leistung besteht darin, dass sie die grammatischen Phänomene des Deutschen $\left(\mathrm{P}_{\text {deutsch i }}\right)$ immer im Vergleich zu entsprechenden Phänomenen in ihrer ganzen typologischen Varianzbreite und insbesondere im Kontrast zu den europäischen Vergleichssprachen erfasst und beschreibt. Das Projekt schlägt die Brücke zur konkreten Anwendung im Bereich des Deutschunterrichts für Ausländer, indem es aus der Konfrontation heraus spezifische Lernschwierigkeiten aufgreift, die das Deutsche für Lerner einer bestimmten Ausgangssprache bei einem spezifischen Problem bietet.

Mit der Forderung nach einer typologisch fundierten Deutsch-Grammatik als Basis für Lehr- und Lernergrammatiken steht dieses Projekt nicht allein. Mit Griesbach 1986 liegt sogar schon ein erster Versuch zu einer typologisch orientierten Referenzgrammatik vor, die aus der praktischen Erfahrung in Sprachunterricht und Spracherwerb resultiert.

Abraham 1991, 135 spricht von einer "typologische(n) DaF-Methode" und zeigt an verschiedenen grammatischen Phänomenen (u.a. Reflexivierung im Deutschen und Slawischen, Aspekt und Diskurspartikeln), dass typologische, insbesondere universalgrammatisch fundierte Kenntnisse über Ausgangs- und Zielsprache entscheidende Hilfestellung beim Verstehen und bei der Korrektur von Interferenzfehlern leisten können.

Handwerker 1995, 203 skizziert als Fernziel „eine typologisch orientierte Grammatik des Deutschen für DaFLernende mit einem Leitfaden zur Umsetzung in einem erklärenden Unterricht“. Gedacht ist dabei ausschließlich an den „Unterricht für Fortgeschrittene, der die kognitiv-analytischen Strategien der Schüler stärken und ausnutzen will“, aber auch an die „Verbesserung der fremdsprachlichen Produktion durch eine 'tiefere' Verarbeitung der neuen Phänomene“.

Auch Fabricius-Hansen 1999, 63f. plädiert aus der Sicht der Auslandsgermanistik für grammatische Beschreibungen des Deutschen "mit einem kontrastiv-typologischen Hintergrund". Diese wiederum können als Basis für mehr didaktisch orientierte Grammatiken dienen, "die von einer individuellen Ausgangssprache ausgehen und somit im engeren Sinne (explizit oder implizit) kontrastiv sind."

c) kulturpolitisch: Förderung des europäischen Sprachbewusstseins

Noch eine dritte Motivation spielt eine Rolle. Es erscheint reizvoll, auf diesem Wege einen grammatischen Beitrag zur kulturellen Identität Europas zu leisten. Bisher wird im europäischen Kontext die Frage der Zukunft der einzelnen Nationalsprachen in erster Linie unter dem Aspekt der kulturell-politischen Geltung diskutiert: So werden Befürchtungen laut, das Englische werde im Zuge der "Globalisierung" die anderen Nationalsprachen nicht nur immer massiver beeinflussen, sondern letztlich sogar im Bereich von Politik, Wirtschaft und Wissenschaft verdrängen. Dabei hat die (deutsche) Sprachwissenschaft bisher nur zögerlich reagiert und, wenn überhaupt, dann mit einseitigem Blick auf die "eigene" Sprache. Vernachlässigt wurde dagegen die Förderung einer Art 'europäischen Sprachbewusstseins', das auf einem besseren Verständnis sprachstruktureller Gemeinsamkeiten und Kontraste 
zwischen europäischen Sprachen beruhen könnte. Die europäische 'Sprachidentität' wird wie die kulturelle Identität der Europäer überhaupt auf dem Prinzip der Vielfalt beruhen, also auf dem Prinzip der Sprachenvielfalt auf der Basis erkannter Gemeinsamkeit. In Europa, so wissen wir, werden auch heute noch mehr als 100 (vom Isländischen bis zum Georgischen oder Tschetschenischen) Sprachen gesprochen (und z.T. auch geschrieben). Diese Multilingualität ist ein Spiegel der kulturellen Vielfalt Europas. Die Antwort des Individuums auf die Multilingualität ist die Mehrsprachigkeit - und was läge näher, als die Sprache der europäischen Nachbarn zu erlernen oder sich zumindest über sie zu informieren. Lexikalische Übereinstimmungen haben z.B. unter dem Stichwort ' Eurolatein' bereits wissenschaftliche Beachtung gefunden. Unser Vorhaben wird die grammatischen Evidenzen offerieren, mit denen vor allem Sprecher des Deutschen, aber auch die Sprecher der Kontrastsprachen, ihr europäisches Sprachbewusstsein untermauern können.

\section{Unterschiede zu kontrastiven Unternehmungen}

Es ist gerade nach dem eben Gesagten klarzustellen, dass GDE keineswegs eine Anhäufung kleiner kontrastiver Grammatiken sein darf; der typologisch differenzierende und das Deutsche fokussierende Blick muss vielmehr in einer anderen Form umgesetzt werden, als dies die traditionellere kontrastive Perspektive nahelegt. Das geplante Unternehmen unterscheidet sich sowohl von der kontrastiven Grammatik als auch von der allgemeinen Sprachtypologie:

Wenn das Ziel der kontrastiven Linguistik "ein umfassender Vergleich zweier Sprachen" ist, der "grundsätzlich nicht gerichtet ist" (König 1996, 32), so gilt dies für das geplante Vorhaben nicht uneingeschränkt. Ziel der kontrastiven Dimension des Vorhabens ist der feinkörnige Vergleich des Deutschen, also der Vergleich im Hinblick auf möglichst viele Variablen oder Varianzparameter/Kontrastdimensionen, mit einer überschaubaren Reihe von Kontrastsprachen, der jedoch im Hinblick auf die Charakterisierung des Deutschen gerichtet ist.

Ein Ziel der allgemeinen Sprachtypologie ist dagegen nach König der systematische Vergleich möglichst "vieler Sprachen entlang weniger Parameter der Variation" (König a.a.O.). Man kann hier auch von "partieller" oder "konstruktionsbezogener Typologie" sprechen (vgl. dazu auch Comrie 1996). ${ }^{4}$ Auch davon unterscheidet sich GDE: In ihrer typologischen Dimension soll sie das Gesamtspektrum möglicher Variation im Hinblick auf

\footnotetext{
4 Einen Überblick über die aktuelle typologische Forschung bieten Croft 1990, Shibatani/Bynon 1995, Stromsdörfer 1995.
} 
ein Phänomen $\mathrm{P}$ insgesamt grob umreißen, im Hinblick auf den Bereich europäischer Sprachen genauer eingrenzen und damit eine Basis für die Profilierung der Kontraste zwischen dem Deutschen und den Kontrastsprachen bzw. des Deutschen selbst bieten.

\section{Theoretischer Zugang, Methodik}

Soweit wie möglich, soll zu jedem Phänomenbereich ein interlingual funktionaler Zugang gewählt werden. Es wird dabei aber nicht behauptet, dass es einen universalen Satz von Funktionen gibt, den jede Sprache nur umsetzt. Dies wäre eine nicht-akzeptable pragmatische Wendung von Chomskys Prinzipien-und-Parameter-Modell.

Ebenso wenig wird angenommen, dass es sinnvoll sei, eine 'Begriffssprache' zu etablieren, die grammatische Funktionen (und Formen) unabhängig von jeder einzelsprachlichen Ausprägung zu erfassen sucht und die für die Kontrastierung als tertium comparationis fungiert.

Gegründet auf Erfahrungen mit der GDS und unter der besonderen Fokussierung des Deutschen ist vielmehr folgender Zugang denkbar:

Als heuristische Basis kann zunächst von ' funktionalen Domänen' (vgl. "functional domain" vgl. Frajzyngier 1999, 126) ausgegangen werden, die bestimmten formal definierten größeren sprachlichen Objektbereichen zugeordnet werden. Hierbei handelt es sich um vortheoretische Konstrukte, die einer ersten sprachübergreifend funktionalen Sortierung dienen sollen. Dem Objektbereich der nominalen Konstruktionen können z.B. die folgenden funktionalen Domänen zugeordnet werden:

- ' Klassifizierung bzw. Sortierung des Referenzbereichs' , z.B. in Personen und NichtPersonen, in Kollektive und Individuen usw.

- 'Referenzkonstitution' bzw. 'Referenzrestriktion' , z.B. durch Attribuierung von Adjektivphrasen, Nominal- und Präpositionalphrasen

- ' Determination' , z.B. durch Artikelsetzung.

Für den Objektbereich des Verbs oder verbaler Konstruktionen ist an funktionale Domänen zu denken wie

- ' Temporalität' , ' Aspektualität' , ' Modalität' , ' Diathese'.

Auf der Satzebene geht es um funktionale Domänen wie

- ' Sachverhaltskonstitution/Partizipation' , ' Sachverhaltsmodifikation' 
Der Ausgangspunkt "größerer Objektbereich" + "zugehörige funktionale Domänen" wird aufgrund folgender Überlegungen gewählt: Es gibt kein universales Raster von Wortklassen und Konstruktionstypen (vgl. dazu Sasse 1993). Diese Ebene "kleiner" Objekte scheidet also für den interlingualen Vergleich zunächst aus. Wohl aber gibt es universale Übereinstimmung in der Existenz einiger weniger syntaktischer oder auch morphologischer Konstrukte, die bestimmten notwendigen kommunikativen Grundfunktionen dienen: Als solche werden genannt: die Referenz (als Grundfunktion nominaler Konstruktionen), die Prädikation (als Grundfunktion verbaler Konstruktionen im Satz) und die Attribution (als Grundfunktion adjektivischer Konstruktionen). Assoziiert mit diesen Grundfunktionen sind aus unserer Sicht die funktionalen Domänen im o.g. Sinne. Sie spalten die Grundfunktionen in eine Reihe von zugehörigen ebenfalls als sprachübergreifend anzusehenden Aspekten auf. Im Unterschied zu den Grundfunktionen selbst dürften die zugeordneten Einzeldomänen jedoch nicht notwendigerweise universalen Status haben.

Relativ $\mathrm{zu}$ solchen formal bestimmten größeren Objektbereichen und den zugehörigen vorläufig abgesteckten funktionalen Domänen werden, wo immer möglich, formale Differenzierungen des Deutschen als eigentlicher methodischer Ausgangspunkt gewählt.

- Dies ist exemplarisch gut möglich auf der Ebene des Wortes, zumal bei Kategorisierungen in den nominalen und verbalen Paradigmen (im Sinne von Eisenberg 1998). So sind also für die Substantive, Adjektive, Determinative, Pronomina die Kategorisierungen 'Genus', 'Kasus', 'Numerus' zu behandeln, für die Verben ' Person' , ' Numerus' , ' Tempus' , ' Modus' und ' Genus Verbi' .

- Im phrasalen Bereich empfiehlt sich ebenfalls ein Ausgehen von im Deutschen vorhandenen Konstruktionstypen als methodische Basis, z.B. Nominalphrase, Relativsatz, Infinitivkonstruktion, Subjunktorsatz usw.

- Auf der Satzebene soll an die GDS-Unterscheidungen zwischen ' kompositionaler Struktur' (Satzgliedebene), ' linearer Struktur' (Konstituentenordnung), ' nicht-kompositionalem Satzmodus' und ' Informationsstruktur' (' Diskurspragmatik' ) angeknüpft werden. Bei der Beschreibung der kompositionalen Struktur muss aber im Interesse des Sprachvergleichs über die GDS-Systematik hinausgegangen werden. Dort sind 11 Komplementklassen definiert, wobei zumindest indirekt partiell auf Kasusunterscheidungen (Akkusativkomplement, Dativkomplement) zurückgegriffen wurde. Diese Klassenbildung muss - ebenso wie eine entsprechende in den Kontrastsprachen, etwa mit dem Instrumentalkomplement des Russischen/Polnischen - abgebildet werden auf eine 
Klassenbildung nach syntaktischen Funktionen (Subjekt, direktes/indirektesObjekt usw.), die einen Vergleich ermöglicht, etwa im Hinblick auf die syntaktische Realisierung von thematischen Rollen.

Die so identifizierten Konstruktionstypen sind neben der formalen Deskription auf ihre systembezogene und ihre konzeptuelle Funktionalität im Rahmen der abgesteckten funktionalen Domäne(n) zu befragen. Erst bei diesem Analyseschritt werden die übereinzelsprachlich nur vage umrissenen konzeptuellen und systemimmanenten Funktionen präzise und auf das Deutsche bezogen ausdifferenziert. Ich erläutere dies am Beispiel des Substantivgenus. Eine erste vororientierende funktionale Bestimmung des Substantivgenus könnte lauten:

"Genus ist eine Klassifizierung des nominalen Lexikons, die semantisch und/oder formal basiert sein kann. Die Klassenzugehörigkeit eines Nomens drückt sich notwendig in der Form seiner Bezugseinheiten aus und kann darüber hinaus auch am Nomen selbst markiert sein." 5

Für das Deutsche kann dann z.B. festgestellt werden, dass die wichtigste ordnungsstiftende Rolle wortbildungsmorphologische Regularitäten spielen, während semantische und auch phonologische Ordnungsdeterminanten weniger bedeutend sind. Man kann dies auch so interpretieren, dass im Deutschen die systembezogene Funktionalität des Genus überwiegt, da eine konzeptuelle oder semantische Funktion des Genus überwiegend nur im Bereich der Personenbezeichnungen (oder der Bezeichnung höherer Lebewesen) vorliegt.

Dieser abgesteckte funktionale Rahmen ist die Vergleichsbasis, nicht ein abstraktes tertium comparationis. Das weitere Vorgehen umfaßt dann sukzessive folgende Fragestellungen:

- Kennt die Kontrastsprache eine grammatikalisierte Differenzierung innerhalb des entsprechenden Objektbereichs, die funktional vergleichbar ist? Wenn ja, arbeite die formalen und funktionalen Gemeinsamkeiten und Unterschiede heraus. Im Beispielfall ist zunächst festzustellen, dass einige der Kontrastsprachen (Ungarisch, Türkisch) kein Substantivgenus haben, andere (die romanischen Sprachen außer Rumänisch) nicht drei Genera, sondern nur zwei und dass z.B. das Englische, das Niederländische und die skandinavischen Sprachen nur reduzierte Unterscheidungen aufweisen. Allerdings darf an dieser Stelle nicht schon die Untersuchung abgeschlossen werden. Selbst wenn das Ungarische oder Türkische kein Genus kennen, ist nicht per se ausgeschlossen, dass nicht

\footnotetext{
${ }^{5}$ vgl. Manuskript Ursula Hoberg "Das Genus des Nomens", S. 3 (IDS Mannheim 2000, Projekt GDE)
} 
eine andere grammatikalisierte Sortierung des nominalen Lexikons vorliegt, die zumindest partiell der funktionalen Bestimmung genügt. ${ }^{6}$

- Kennt die Kontrastsprache keine entsprechende grammatikalisierte Differenzierung, nenne gegegebenenfalls lexikalische oder periphrastische Verfahren, die funktionale Berührungspunkte aufweisen. Man kann hier beim Substantivgenus an Wortbildungsverfahren denken, mit deren Hilfe z.B. aus Bezeichnungen für männliche Personen (Berufsbezeichnungen) Bezeichnungen für weibliche Personen abgeleitet werden. $^{7}$

Zwischen diesen beiden Möglichkeiten sollte aber möglichst klar unterschieden werden. Werden die Unterschiede nicht beachtet, gelangt man $\mathrm{zu}$ 'transgrammatischen' Kategorisierungen, bei denen der Unterschied zwischen Grammatik und Lexikon nicht beachtet wird, wie eben im Beispiel der Sexusdifferenzierung durch Genus und der Sexusdifferenzierung durch Wortbildung. Eine ähnliche transgrammatische Kategorisierung liegt vor, wenn der Unterschied z.B. zwischen dem Passiv und anderen semantisch ' inaktiven' oder "geschehensbezogenen" (vgl. Engel u. a. 1999, 659) Konstruktionen, die aber eher nicht als ein eigenes Genus Verbi, sondern als lexikalische Konversen oder als syntaktische Fügungen einzuschätzen sind, nicht klar genug herausgearbeitet wird. Der Fokus liegt nicht beim Aufweisen funktionaler (Teil-)Äquivalenzen, sondern auf dem je spezifischen Verhältnis zwischen grammatischer Form und Funktion. In einem nächsten Schritt ist zu prüfen:

- Sind im gegebenen Objektbereich in den Kontrastsprachen Kategorisierungen vorhanden, die es im Deutschen nicht gibt? Wenn ja, beschreibe sie bei der entsprechenden Kontrastsprache in Grundzügen und vermerke beim Deutschen das Nicht-Vorhandensein sowie ggf. entsprechende Konstruktionen mit funktionalen Berührungspunkten. Ein typisches Beispiel für diesen Fall ist die verbale Kategorisierung ' Aspekt', die im Deutschen im Gegensatz vor allem zu den slawischen Sprachen nicht vorhanden ist.

Bei Anwendung der geschilderten Analyseschritte auf alle Kontrastsprachen zu einem formal und funktional eingegrenzten Phänomen soll sich ein Raster von funktionalen und formalen Merkmalen ergeben, das sowohl die interlingual konstanten, gemeinsamen Eigenschaften (auf funktionaler wie auf formaler Seite) enthält als auch die von Sprache zu Sprache variierenden.

\footnotetext{
${ }^{6}$ So gibt es im Türkischen zumindest einen Reflex der Unterscheidung von personenbezeichnenen und nichtpersonenbezeichnenen Substantiven beim korrespondierenden Verb (vgl. Kornfilt 1997, 271).

So kann im Ungarischen bei einigen Status- oder Berufsbezeichnungen durch Suffigierung mit nö 'Frau' verfahren werden: király - kiranynő 'König - Königin', orvos - orvosnő 'Arzt - Ärztin'.
} 
Als Umsetzung dieses Programms in dem geplanten Projekt kann das Verfahren einer 'fortschreitenden kontrastiven Form- und Funktionsdifferenzierung' dienen. ${ }^{8}$ Das bedeutet, dass die Beschreibung der Formmöglichkeiten (Morphologie, Syntax, Distribution) und der Funktion einer grammatischen Kategorie oder eines Konstruktionstyps, die vorläufig für das Deutsche festgelegt wurde, beim Vergleich mit dem entsprechenden Phänomen in einer bestimmten Kontrastsprache immer dann in einzelne verschiedene 'Muster' (Formseite) bzw. 'Verwendungen' (Funktionsseite) zerlegt wird, wenn, global gesehen, partielle Überlappung vorliegt. Ergebnis einer solchen Zerlegung sind Einzelmuster/Einzelverwendungen, in denen Deutsch und die Kontrastsprache (weitgehend) übereinstimmen, neben solchen, in denen sie sich unterscheiden. Der Weg führt also von der globalen partiellen Ähnlichkeit zu der muster- bzw. verwendungsspezifischen Identität/Ähnlichkeit und Verschiedenheit. Ein wichtiger methodischer Schritt, der sich auch in der Präsentation dann niederschlagen soll, ist eben die Isolierung invarianter Merkmale, die als notwendig relativ zu dem Konstruktionstyp und der Menge der Vergleichssprachen betrachtet werden können, gegenüber den Parametern der Varianz, die jeweils durch interlingual nicht-notwendige, für die Einzelsprache jedoch wesentliche Merkmalsausprägungen belegt werden.

Das Vorgehen kann in folgendem Ablaufplan festgehalten werden:

1. Einem formal bestimmten "größeren" Objektbereich $\mathrm{O}_{\mathrm{i}}$ (z.B. einer in allen Vergleichssprachen existenten Wortklasse, einem in allen Vergleichssprachen bezeugten "größeren" Konstruktionstyp wie 'Nominale', 'Verbale') werden zu heuristischen Zwecken 'funktionale Domänen' $\mathrm{F}_{1, \ldots, \mathrm{n}}$ zugeordnet.

2. Formale Kategorisierungen / Konstruktionstypen $\mathrm{K}_{\mathrm{i}}$ des Deutschen aus $\mathrm{O}_{\mathrm{i}}$ dienen als methodischer Ausgangspunkt. Es erfolgt eine vorläufige formale und funktionale Ausdifferenzierung bezogen auf das Deutsche. Dabei ist davon auszugehen, daß $\mathrm{K}_{\mathrm{i}}$ funktional mindestens eine funktionale Domäne $F_{j}$ aus der $O_{i}$ zugeordneten Menge $F_{1, \ldots, n}$ abdeckt. Für diese funktionale Domäne $F_{j}$ erfolgt eine genaue Spezifikation in Form von Einzelmustern und Einzelverwendungen im Deutschen.

3. Grammatikalisierte Phänomene in den Kontrastsprachen, vorzugsweise aus Objektbereich $\mathrm{O}_{\mathrm{i}}$ (gegebenfalls auch aus einem anderen Objektbereich $\mathrm{O}_{\mathrm{j}}$ ), mit zum Deutschen vergleichbarer bzw. sich überschneidender Funktion (bezogen auf $F_{j}$ ) werden gegenübergestellt. Die formalen Merkmale und die Einzelmuster/Einzelverwendungen

\footnotetext{
${ }^{8}$ Vgl. auch das ähnliche Konzept der "comparative form-function mappings" bei Kemmer 1993, 7.
} 
müssen auch bezüglich der Kontrastsprachen vorläufig isoliert werden. Die "endgültige" Festschreibung von Einzelmustern und Einzelverwendungen in den Vergleichssprachen erfolgt erst nach sorgfältiger Abgleichung der Befunde. Erst dieser interlinguale Vergleich zeigt (unter Berücksichtigung des allgemeintypologischen Hintergrundes), wo Grenzen zwischen Mustern und Funktionen anzusetzen sind. Ein gutes Beispiel findet sich bei den Personalpronomina. Das nicht-referentielle "neutrale" Personalpronomen der 3. Person (dt. es) hat Parallelen in allen anderen germanischen Sprachen sowie im Französischen, also ausschließlich in Sprachen ohne das "Pro-drop-Phänomen". Der interlinguale Vergleich zeigt, dass es in zwei Konstruktionstypen verwendet wird: in "unpersönlichen" Konstruktionen (z.B. bei Witterungsverben: dt. es regnet) in der Funktion eines formalen Argumentes oder 'Quasi-Argumentes' und durch strukturellen Vorverweis zur "Rhematisierung" von Konstituenten. Bei Letzterem schafft die Setzung des neutralen Pronomens die Möglichkeit, z.B. das Subjekt in den rhematischen Bereich am Satzende zu versetzen (dt. Es kamen drei nette Jungs). Auffällig ist nun, dass im Dänischen, Niederländischen und Englischen bei einigen Untertypen, wo die übrigen Vergleichssprachen das neutrale Pronomen verwenden, ein lokales Adverb (engl. there) gebraucht wird. Dies betrifft zum einen unpersönliche Konstruktionen mit Existenzverben (engl. There is a unicorn in the garden), zum anderen aber auch intransitive Geschehensverben, wenn das indefinite Subjekt nach rechts versetzt und damit im rhematischen Bereich präsentiert werden soll (engl. There came three nice boys). Man kann daraus den Schluss ziehen, dass die Einzelmuster 'Existenzverben' und 'Rechtsversetzung des Subjekts' in den drei Sprachen mit Adverbgebrauch einer gemeinsamen Einzelfunktion 'Lokalisierung von Partizipanten in einer Situation' zugeordnet sind, während die entsprechenden Muster in den übrigen Sprachen den Funktionen 'Lieferung eines Quasi-Argumentes' und 'struktureller Vorverweis durch Platzhalter' zugeordnet sind.

4. Aufgrund der in 3. geschilderten auf alle Kontrastsprachen angewendete fortschreitenden Form-Funktionsdifferenzierung wird ein Raster aller invarianten Merkmale auf der Formund der Funktionsseite sowie aller zwischen den Vergleichssprachen divergierenden Eigenschaften (relativ zur jeweiligen Kategorisierung und der einschlägigen funktionalen Domäne $F_{j}$ ) erstellt. Die divergierenden Merkmale werden nach Parametern der Varianz geordnet. $^{9}$

\footnotetext{
9 Zu einer vergleichbaren Vorgehensweise bei der interlingualen Isolierung von Verwendungen ("uses") grammatischer Kategorien in der Sprachtypologie vgl. Haspelmath 1997, 60 f, der sich seinerseits auf Ferguson 1970 und die Praxis in der
} 


\section{Darstellungsformat und Konkretisierung: Beispiel Relativsatz}

Bei einem sprachvergleichenden Projekt der beschriebenen Art wird eine große Informationsmenge auf neuartige und komplexe Weise miteinander vernetzt. Es wird für die Nutzbarkeit ganz wesentlich darauf ankommen, den Zugriff auf die Informationen zu erleichtern. Dazu ist eine konsequente, transparente und möglichst einheitliche Struktur, in der die Informationen dargeboten werden, die erste Voraussetzung. Die vorzusehende Struktur muss den vier Grundpfeilern des Vorhabens gerecht werden: (1) funktional ausgerichtete typologische Orientierung zu einem Phänomen, (2) Darstellung des Phänomens in den Kontrastsprachen als Folie, (3) Darstellung des Phänomens im Deutschen als Fokus (4) Bezug zum Anwendungsbereich Deutsch als Fremdsprache. Die in Abschnitt 5 erläuterte Methodologie findet dabei folgenden Niederschlag: Sprachübergreifende bzw. invariante Aspekte des Phänomens werden durch Punkt (1) erfasst; sprachspezifische Unterschiede, die nach Parametern geordnet sind, werden bei Punkt (2) und (3) systematisch dargestellt.

Jedes Thema, z.B. das Thema Relativsatz, soll daher in vier entsprechenden Bausteinen abgehandelt werden. Diese sind:

1. Funktionale und typologische Charakterisierung

Hier soll ein typologischer Gesamtüberblick zu einem Phänomen auch unter Einbeziehung außereuropäischer Sprachen gegeben werden. Dabei werden die Varianzparameter isoliert.

2. Die Belegung derVarianzparameter in den europäischen Sprachen

Die Binnengliederung ergibt sich durch Abruf der einzelnen Parameter, nicht etwa nach Sprachen. Bei jedem Parameter ist auf die Kontrastsprachen einzugehen, andere europäische Sprachen können hinzugenommen werden. Nach Möglichkeit werden tabellarische Übersichten gegeben.

3. Das Deutsche

Auch hier ergibt sich eine Binnengliederung durch den Abruf der Parameter. Die Behandlung im Deutschen soll sehr viel eingehender sein als die der Kontrastsprachen.

4. Lernschwierigkeiten bezüglich bestimmter Ausgangssprachen

Hier sollen relativ zu bestimmten L1 aus der Menge der Kontrastsprachen Schwierigkeiten beim Erlernen des Deutschen als L2 erörtert werden. Die Erörterung soll grundsätzlich auf die Belegung von Varianzparametern bezogen werden und ganz gezielt an bestimmte Stellen in Baustein 2 und 3 anknüpfen.

Das Konzept des Varianzparameters spielt also auch darstellungstechnisch eine wichtige Rolle. Um etwas näher zu erläutern, wie solche Varianzparameter aussehen, sollen einige derjenigen, die für das Relativsyntagma (bzw. den Relativsatz) herausgearbeitet wurden, hier präsentiert werden.

typologischen Beschreibung von Kategorien wie Tempus, Aspekt, Modus und Genus Verbi in den vergangenen zwanzig Jahren beruft, sowie Kemmer 1993, 7. 


\section{Varianzparameter für Relativsyntagmen ${ }^{10}$ :}

1. Relativsatz und Relativinfinit: Verfügt eine Sprache über Relativsätze oder Relativinfinite? Wenn sie beide Formen kennt, welche ist dominant?

Das Türkische ist unter den Vergleichssprachen die einzige, bei der infinite Relativkonstruktionen dominant sind.

2. Subordination: Wird Subordination überhaupt syntaktisch ausgedrückt? Wenn ja, wird sie durch einen Subordinator oder affixal ausgedrückt? Ist der Subordinator ein Relativpronomen/Relativadverb oder eine Relativpartikel? Von welchen weiteren Mittel der Subordination, z.B. Nebensatzstellung, wird Gebrauch gemacht?

Im Japanischen z. B. gibt es keinen syntaktischen Ausdruck für die Subordination von Relativsyntagmen. In den europäischen Sprachen hingegen überwiegen Relativsyntagmen mit einem Subordinator; affixale Subordination gibt es im Baskischen. Im Englischen und den skandinavischen Sprachen kann in bestimmten Fällen die Setzung des Subordinators unterbleiben. Viele europäische Sprachen haben Relativpronomina, also veränderliche Ausdrücke, die mit ihrem Bezugswort in Genus und Numerus korrespondieren und die durch ihren Kasus die syntaktische Funktion anzeigen. Typische Beispiele sind die deutschen und die polnischen Relativpronomina. Das engl. that und das dän./norw./schwed. som sind Beispiele für Relativpartikeln.

3. Syntaktische Relationen: Ist das Relativsyntagma adnominal (mit externem Nukleus und syntaktisch integriert), dabei prä- oder postnominal? Oder ist es zirkumnominal (mit internem Nukleus und syntaktisch integriert)?

Im europäischen Raum gibt es nur adnominale Relativsyntagmen. Zirkumnominale Konstruktionen sind vor allem in amerikanischen Sprachen (z.B. Navaho) vertreten. Bei zirkumnominalen Relativsyntagmen ist das Bezugsnomen Teil des Relativsyntagmas. Wir können das im Deutschen etwa durch folgendes Beispiel imitieren: *Ich werde, welchen Weg du gehst, auch gehen. Das europäische Relativsyntagma ist überwiegend postnominal. Beispiele für pränominale Relativsyntagmen liefern Japanisch oder Mandarin, im europäischen Raum Türkisch und Baskisch.

4. Zugänglichkeitshierarchie für syntaktische Relationen: Welche syntaktischen Funktionen sind in einer Sprache für den syntaktischen Repräsentanten x' der semantischen Leerstelle im Relativsyntagma zugelassen?

In manchen Sprachen können nur Ausdrücke in zentralen syntaktischen Funktionen "relativiert" werden. Die syntaktischen Funktionen können dabei in einer universal gültigen Zugänglichkeitshierarchie (nach Keenan/Comrie 1977) angeordnet werden. Im Madegassischen ist z.B. nur die Subjektsfunktion zugänglich für Relativierung. Die meisten europäischen Sprachen verfügen über einen vergleichsweise großen Ausschnitt aus der Zugänglichkeitshierarchie. Nur das Komplement von Vergleichskonstruktionen ist in

\footnotetext{
${ }^{10}$ Insgesamt werden in der Pilotstudie acht Varianzparameter erörtert. Neben den im Text diskutierten sind noch zu nennen: 'Pronominalisierung oder Lückenbildung', 'Relativierbarkeit mittelbarer Konstituenten', 'freier
} 
vielen europäischen Sprachen nicht relativierbar (vgl. aber engl.: the man (who) I am taller than gegenüber dt. *der Mann als welcher ich größer bin)

Eines der Ergebnisse einer vergleichenden Studie zum Relativsysntagma verdient im Hinblick auf die oben genannte kulturpolitische Zielsetzung des Unternehmens Beachtung: Nur in Europa gibt es den Typ des Relativsatzes, der postnominal gestellt wird, und der von einem Relativpronomen eingeleitet wird, das mit dem Bezugswort in Genus und Numerus übereinstimmt (dt. der Mann, der - die Frau, die; lat. vir qui - femina quae; poln. mężczyzna który - kobieta która) und das gleichzeitig die syntaktische Funktion im Relativsatz verdeutlicht. Zwar ist dies nicht der einzige in Europa vorkommende Typ, wie die Varianparameter zeigen, in anderen Weltregionen gibt es ihn aber gar nicht.

\section{Forschungspraktisches Vorgehen}

Das Projekt steckt wie gesagt noch in den Kinderschuhen; es müssen neue Mitarbeiter hinzugewonnen werden. Noch ist auch unklar, welche Teile diese Grammatik umfassen soll, ob es überhaupt ein in sich geschlossenes Werk oder einzelne Studien ergibt. Fest steht im Moment, dass wir mit dem nominalen Bereich fortfahren - wahrscheinlich wird es dazu auch eine erste Publikation geben. Ein wichtiger abschließender Gesichtspunkt ist die Artikulation des Wunsches nach Kooperation: Wir wünschen uns die Zusammenarbeit mit Vertretern der einzelnen Kontrastsprachen, es kann sich dabei um Polonisten bzw. Hispanisten usw. oder polnische bzw. spanische Germanisten handeln oder aber um kontrastiv und typologisch arbeitende Sprachwissenschaftler mit entsprechenden Sprachkenntnissen.

\section{Literatur:}

Abraham, Werner (1991): Die Logik der Lehre des 'Deutschen als Fremdsprache (DaF)'. Papiere zur Linguistik Nr. 44/45 (Heft 1/2). 131-157.

Abraham, Werner (1994): Deutsche Syntax im Sprachenvergleich. Grundlegung einer typologischen Syntax des Deutschen. Tübingen: Narr. (= Studien zur deutschen Grammatik 41)

Cartegena, Nelson/Gauger, Hans-Martin (1989): Vergleichende Grammatik SpanischDeutsch. Mannheim: Duden. (= Duden Sonderreihe vergleichende Grammatiken 2)

Comrie, Bernard (1996): Sprache und Sprachen: Universalien und Typologie. In: Lang, Ewald /Zifonun, Gisela (eds.) (1996): Deutsch - typologisch. Berlin/New York: de Gruyter. 16-30.

Croft, William (1990): Typology and Universals. Cambridge: Cambridge University Press.

Eisenberg, Peter (1998): Grundriss der deutschen Grammatik. Das Wort. Stuttgart/Weimar: J. B. Metzler. 
Engel,Ulrich/Mrazović,Pavica (1986): Kontrastive Grammatik deutsch-serbokratisch. München: Sagner. (= Sagners slavistische Sammlung 10)

Engel, Ulrich (1991): Deutsche Grammatik. Heidelberg: Groos.

Engel, Ulrich/Isbaşescu, Mihai/Stanescu, Speranta/Nicolae, Octavian (1993): Kontrastive Grammatik deutsch-rumänisch. Heidelberg: Groos.

Engel, Ulrich et al. (1999): Deutsch-polnische kontrastive Grammatik. 2 Bde. Heidelberg: Groos.

Fabricius-Hansen, Cathrine (1999): Welchen Bedarf hat die Auslandsgermanistik? In: Stickel, Gerhard (ed.) (1999): Sprache - Sprachwissenschaft - Öffentlichkeit. Berlin/New York: de Gruyter. (= Jahrbuch 1998 Institut für Deutsche Sprache).

Ferguson, Charles A. (1970): Grammatical Categories in Data Collection. Working Papers in Language Universals 4.

Frajzyngier, Zygmunt (1999): Domains of point of view and coreferentiality: System interaction approach to the study of reflexives. In: Frajzyngier, Zygmunt/Curl, Traci S. (eds.) (1999): Reflexives. Forms and Functions. Amsterdam/Philadelphia: John Benjamins. 125-153 (= Typological studies in language 40).

Glinz, Hans (1994): Grammatiken im Vergleich. Deutsch - Französisch - Englisch - Latein. Tübingen: Niemeyer.

Griesbach, Heinz (1986): Neue deutsche Grammatik. Berlin/München: Langenscheidt.

Handwerker, Brigitte (1995): Zur Relevanz eines typologischen Fragenkatalogs für Lernerund Lehrergrammatiken. In: Handwerker, Brigitte: Fremde Sprache Deutsch. Grammatische Beschreibung - Erwerbsverläufe - Lehrmethodik. Tübingen: Narr. 203224.

Haspelmath, Martin (1997): Indefinite Pronouns. Oxford: Clarendon Press. (Oxford Studies in Typology and Linguistic Theory).

Hawkins, John (1986): A Comparative Typology of English and German. Unifying the Contrasts. London: Croom Helm.

Keenan, Edward L./Comrie, Bernard (1977): Noun phrase accessibility and universal grammar. In: Linguistic Inquiry. Cambridge, MA.: MIT Press. 63-99.

Kemmer, Suzanne (1993): The Middle Voice. Amsterdam/Philadelphia: John Benjamins. (=Typological studies in language 23).

Kiefer, Ferenc (1987): The cases of Hungarian nouns In: Acta linguistica Hungarica (37). 93101.

König, Ekkehard (1996): Kontrastive Grammatik und Typologie. In: Lang, Ewald /Zifonun, Gisela (eds.) (1996): Deutsch - typologisch. Berlin/New York: de Gruyter. 31-54.

Kornfilt, Jaklin (1997): Turkish. London/New York: Routledge.(Descriptive Grammars).

Lang, Ewald (1996): Das Deutsche im typologischen Spektrum. Einführung in den Band. In: Lang, Ewald /Zifonun, Gisela (eds.) (1996): Deutsch - typologisch. Berlin/New York: de Gruyter. 7-15.

Lotz, János (1939): Das ungarische Sprachsystem. Stockholm: Ungarisches Institut Stockholm.

Sasse, Hans-Jürgen (1993): Syntactic Categories and Subcategories. In: Jacobs, Joachim/von Stechow, Arnim/Sternefeld, Wolfgang/Vennemann, Theo (eds.) (1993): Syntax. Ein 
internationales Handbuch zeitgenössischer Forschung. Berlin/New York: Walter de Gruyter. (1.Halbband). 646-686.

Shibatani, Masayoshi/Bynon, Theodora (eds.) (1995): Approaches to Language Typology.Oxford/New York: Clarendon Press.

Strömsdörfer, Christian/Vennemann, Theo (1995): Ziele der syntaktischen Typologie. In: Jacobs, Joachim/Stechow von, Arnim/Sternefeld, Wolfgang/Vennemann, Theo (eds.) (1995): Syntax. Ein internationales Handbuch zeitgenössischer Forschung. Berlin/New York: Walter de Gruyter. 1031-1043.

Willems, Dominique (1999): Pour une terminologie grammaticale européenne. Défense et illustration. In: Béguelin, Marie-José/de Pietro, Jean-Francois/Näf, Anton (eds.) (1999): La terminologie grammaticale à l'école: perspectives interlinguistiques. Neuchatel. 129-142. (= travaux neuchatelois de linguistique 31).

Zemb, Jean-Marie (1978): Vergleichende Grammatik Französisch-Deutsch. Mannheim: Bibliographisches Institut. (= Duden Sonderreihe vergleichende Grammatiken 1)

Zifonun, Gisela/Hoffmann, Ludger/Strecker, Bruno u.a. (1997): Grammatik der deutschen Sprache. Berlin/New York: De Gruyter. (= Schriften des Instituts für deutsche Sprache 7) (= GDS) 


\section{Nachtrag 3.1.2001:}

Bei der Sitzung des "Wissenschaftlichen Beirats" des IDS am 10.11.2000 wurden im Hinblick auf das Projekt GDE u.a. folgende Empfehlungen formuliert:

Kontrastsprachen: Eine Beschränkung auf nur vier Kern-Kontrastsprachen (Englisch, Französisch, Polnisch, Ungarisch) erscheint sinnvoll. Im Gegenzug soll mit dem "Ring" weiterer europäischer Kontrastsprachen besonders flexibel umgegangen werden. Das heißt, wo immer es sich vom Phänomenbereich her anbietet, sollen weitere Kontrastsprachen herangezogen werden.

Publikationsform: Es soll eine dreistufige Publikationsform angestrebt werden: Einzelstudien, Bereichsmonographien (z.B. "Der nominale Bereich"), Übersichtswerk (gedacht ist an einen Einbänder als Extrakt aus den Vorpublikationen). Bereits für die Einzelstudien soll eine möglichst einheitliche Darstellungsform vereinbart werden (siehe Abschnitt 6 des vorliegenden Aufsatzes).

Anwendungsbezug: Der Anwendungsbezug (DaF-Bereich) wird grundsätzlich begrüßt. Da aber kaum verlässliche Daten $\mathrm{zu}$ konkreten Lernschwierigkeiten bei Lernern mit verschiedenen Ausgangssprachen zur Verfügung stehen, sollte Baustein 4. (vgl. Abschnitt 6) eher zurückgestellt werden. 